\title{
Self-heating in small mesa structures made of intrinsic Josephson junctions in BSCCO
}

$\operatorname{AUTHOR}(\mathrm{S})$ :

Suzuki, M; Yamada, Y; Tajitsu, E; Kojima, S

\section{CITATION:}

Suzuki, M ...[et al]. Self-heating in small mesa structures made of intrinsic Josephson junctions in BSCCO. IEEE TRANSACTIONS ON APPLIED SUPERCONDUCTIVITY 2007, 17(2): 594-597

\section{ISSUE DATE:}

2007-06

URL:

http://hdl.handle.net/2433/50219

\section{RIGHT:}

(c)2007 IEEE. Personal use of this material is permitted. However, permission to reprint/republish this material for advertising or promotional purposes or for creating new collective works for resale or redistribution to servers or lists, or to reuse any copyrighted component of this work in other works must be obtained from the IEEE. 


\title{
Self-Heating in Small Mesa Structures Made of Intrinsic Josephson Junctions in BSCCO
}

\author{
Minoru Suzuki, Yoshiharu Yamada, Eriko Tajitsu, and Shintaro Kojima
}

\begin{abstract}
We present detailed numerical analysis on the temperature rise due to self-heating for a small mesa in interlayer tunneling spectroscopy based on the thermal diffusion equation using temperature dependent specific heat and thermal conductivity data. In the analysis, a BSCCO square mesa typically $10 \mu \mathrm{m}$ wide and $15 \mathrm{~nm}$ thick with the $c$-axis resistivity of $10 \Omega \mathrm{cm}$ is in close contact with the $350 \mathrm{~nm}$ Au electrode layer on the top. It is found that when a quasiparticle current of $20 \mathrm{~mA}$ is injected, which is a typical value at the superconducting gap voltage, the temperature rise due to self-heating is $3.8 \mathrm{~K}$ at $60 \mathrm{~ns}$ from the pulse rise and $4.6 \mathrm{~K}$ at $500 \mathrm{~ns}$ when the base temperature is $10 \mathrm{~K}$. When the current is $50 \mathrm{~mA}$, the temperature rise is $18 \mathrm{~K}$ at $60 \mathrm{~ns}$ and $22 \mathrm{~K}$ at $500 \mathrm{~ns}$, indicating a significant heating near the edge of the voltage range, where the pseudogap is observed in short-pulse tunneling experiments.
\end{abstract}

Index Terms-Bi-Sr-Ca-Cu-O, high-temperature superconductors, intrinsic Josephson junctions, mesa structure, quasiparticle current, self-heating.

\section{INTRODUCTION}

$\mathbf{T}$ HE intrinsic Josephson junctions (IJJ) [1], [2] naturally built in the layered crystal structure of some high- $T_{c}$ superconductors attract interest from various points of view [3] including the interlayer tunneling spectroscopy (ITS) [4]-[6]. Since the IJJ are closely stacked with a separation of a few $\mathrm{nm}$, the spectroscopy experiments are always accompanied by Joule heating due to self-injection of quasiparticle current, i.e., the self-heating. When the self-heating is significant, the spectroscopy experiments lead to incorrect results. In an extreme case, superconductivity is extinguished by the self-heating. In order to circumvent this problem, a short-pulse technique is employed to suppress the heating to a reasonable order [4], [7]. However, a finite temperature rise always results at the IJJ in the voltage state even in such a short-pulse experiment. It is therefore important to estimate the extent of the temperature rise in such a short-pulse tunneling spectroscopy experiment.

Usually, the temperature rise is a transient response so that direct measurement of the temperature at the IJJ position is difficult. Since there have been only a few reports on the detailed

Manuscript received August 29, 2006. This work was supported in part by 21st Century COE Program Grand of Center of Excellence for Research and Education of Fundamental Technologies in Electrical and Electronic Engineering from the Ministry of Education, Culture, Sports, Science and Technology (MEXT), Japan, and by a Grant-In-Aid for Scientific Research from MEXT.

M. Suzuki is with the Department of Electronic Science and Engineering, Kyoto University, Nishikyo-ku, Kyoto 615-8510, Japan (e-mail: suzuki@ kuee. kyoto-u.ac.jp).

Y. Yamada, E. Tajitsu, and S. Kojima are with Department of Electronic Science and Engineering, Kyoto University, Kyoto 615-8510, Japan.

Digital Object Identifier 10.1109/TASC.2007.898706 numerical calculation of the temperature rise [8], a crude model [9] or an extreme example [10] is often quoted as a general estimate. These models lead to a significant overestimate in the case of short-pulse technique and are misleading.

In the present study, we have carried out detailed numerical calculation of the self-heating by the finite difference time domain method based on Fourier's law of heat conduction for a small mesa structure made of $\mathrm{Bi}_{2} \mathrm{Sr}_{2} \mathrm{CaCu}_{2} \mathrm{O}_{8+\delta}$ (BSCCO) employed in the short-pulse interlayer tunneling spectroscopy experiments [4], [5], [11], [12]. In the analysis, a BSCCO mesa $10 \mu \mathrm{m}$ wide in square side and $15 \mathrm{~nm}$ thick with the c-axis resistivity of $10 \Omega \mathrm{cm}$ is in close contact with the $350 \mathrm{~nm}$ thick $\mathrm{Au}$ electrode layer on the top. It is found that when the quasiparticle current of $20 \mathrm{~mA}$ is injected, which is typically thought to correspond to the superconducting gap voltage for slightly overdoped $\mathrm{BSCCO}$, the temperature rise at $10 \mathrm{~K}$ is $3.8 \mathrm{~K}$ at $60 \mathrm{~ns}$ from the pulse rise and $4.6 \mathrm{~K}$ at $500 \mathrm{~ns}$. When the quasiparticle current is $50 \mathrm{~mA}$, the temperature rise is $18 \mathrm{~K}$ at $60 \mathrm{~ns}$ and $22 \mathrm{~K}$ at 500 $\mathrm{ns}$, indicating a significant heating near the edge of the voltage range in the short-pulse experiments [4], [5]. When the square mesa is $1 \mu \mathrm{m}$ on a side with the injection current at the same density, the temperature rise is less than $1.2 \mathrm{~K}$ both at 60 and $500 \mathrm{~ns}$. The analysis also shows that the temperature rise dominantly depends on the local temperature rise in the Au electrode just above the mesa, so that the self-heating is significantly suppressed as the mesa size is reduced from $10 \mu \mathrm{m}$ to $1 \mu \mathrm{m}$ and the input power is decreased accordingly.

\section{Model ANd CALCUlation}

A model for the numerical calculation is a typical sample employed in the short-pulse ITS [12], as depicted in Fig. 1. The surface of the sample is in contact with flowing $\mathrm{He}$ gas at a pressure of 100 Torr. The mesa is located beneath the Au electrode and sits on the BSCCO single crystal pedestal. The main heat flow channel is via the Au electrode, which has a high thermal conductivity of $2800 \mathrm{~W} / \mathrm{mK}$ at $10 \mathrm{~K}$. In order to evaluate the spatial variation of the temperature, the materials are partitioned to small cells. The Au cell and the BSCCO cell (under-mesa) both in contact with the mesa are further divided to smaller cells. The dimensions of each component and the cells are summarized in Table I. The dimensions other than the mesa size are fixed irrespective of mesa sizes.

The differential equation to be solved is

$$
c_{p} \rho \frac{\partial T}{\partial t}+c_{p} \rho v_{x} \frac{\partial T}{\partial x}+c_{p} \rho v_{y} \frac{\partial T}{\partial y}=\nabla(\tilde{\lambda} \nabla T)+q(t),
$$

where $T$ is the temperature, $c_{p}$ is the specific heat, $\rho$ is the density, $\tilde{\lambda}$ is the anisotropic thermal conductivity tensor, and $q(t)$ is 
(a)

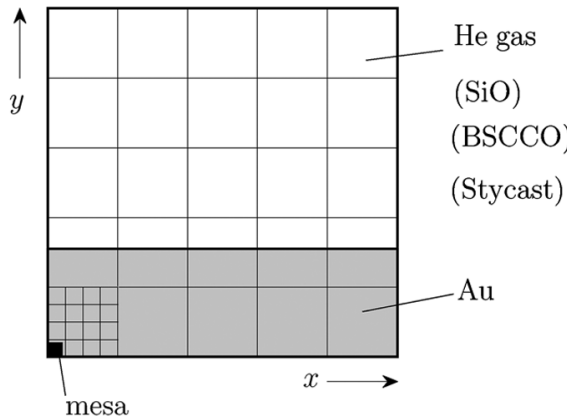

(b)

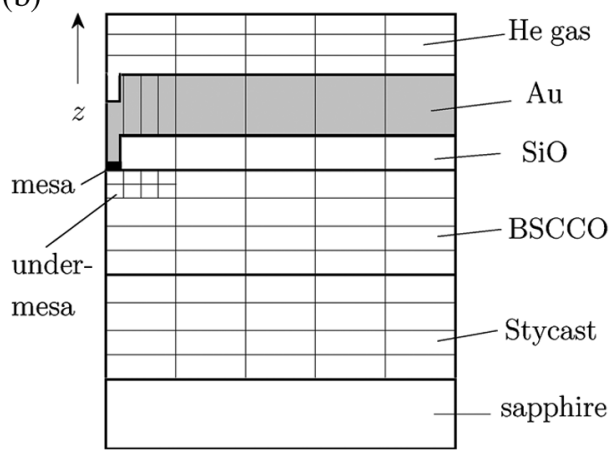

Fig. 1. (a) Plan view and (b) cross sectional view of a small mesa structure as a model for the calculation.

TABLE I

Dimensions of the MESA StRUCTURE USED FoR THE CALCULATION

\begin{tabular}{llll}
\hline & $x$ size $(\mu \mathrm{m})$ & $y$ size $(\mu \mathrm{m})$ & $z$ size $(\mu \mathrm{m})$ \\
\hline mesa & 10 & 10 & 0.015 \\
mesa cell & 10 & 10 & 0.003 \\
BSCCO pedestal & 1000 & 1000 & 40 \\
BSCCO cell & 38.5 & 38.5 & 5.71 \\
under-mesa & 5.5 & 5.5 & 0.381 \\
Au electrode & 1000 & 100 & 0.35 \\
Au subcell & 2.56 & 2.56 & 0.35 \\
SiO layer & 1000 & 1000 & 0.25 \\
SiO cell & 38.5 & 38.5 & 0.25 \\
Stycast layer & 1000 & 1000 & 20 \\
Stycast cell & 38.5 & 38.5 & 4 \\
He gas layer & 1000 & 1000 & 20 \\
He cell & 38.5 & 38.5 & 2 \\
\hline
\end{tabular}

the heat source due to the self-heating. We employed $T$-dependent physical parameters except for $\mathrm{SiO}$ and Stycast (an epoxy resin used as a glue), in which the thermal properties are not known sufficiently. In particular, the thermal conductivity of $\mathrm{SiO}$ is unknown, so we employed a value of $1 \mathrm{~W} / \mathrm{mK}$, which is an intermediate between fused $\mathrm{SiO}_{2}(0.69 \mathrm{~W} / \mathrm{mK})$ and quartz $(39 \mathrm{~W} / \mathrm{mK})$. As seen below, the temperature rise is determined mainly by the BSCCO mesa and the Au electrode. Therefore, the thermal parameters for other materials are less important; they are used to calculate the heat flow from the Au electrode to the BSCCO pedestal. Important physical parameters are listed in Table II and the $T$-dependence of some of them are depicted in Fig. 2 to Fig. 4. The $c_{p}$ data for BSCCO was originally obtained by Loram et al. [13] and the $\lambda_{a b}$ and $\lambda_{c}$ data were measured originally by Crommie and Zettl [14]. For convenience, the units are converted to the SI units in Figs. 2-4.
TABLE II

Thermal CONDUCTIVITY, HeAT CAPACITY, AND DENSITY FOR Au, BSCCO, SiO, STYCAST, AND 100 Torr He AT $10 \mathrm{~K}$

\begin{tabular}{lccc}
\hline Material & $\begin{array}{c}\text { specific heat } \\
(\mathrm{J} / \mathrm{kg} \mathrm{K})\end{array}$ & $\begin{array}{c}\text { density } \\
\left(\mathrm{kg} / \mathrm{m}^{3}\right)\end{array}$ & $\begin{array}{c}\text { thermal conductivity } \\
(\mathrm{W} / \mathrm{m} \mathrm{K})\end{array}$ \\
$\mathrm{Au}$ & 13.7 & $1.93 \times 10^{4}$ & 2800 \\
$\mathrm{BSCCO}(\perp c)$ & 2.82 & $6.814 \times 10^{3}$ & 2.03 \\
$\mathrm{BSCCO}(\| c)$ & & & 0.24 \\
$\mathrm{SiO}$ & 840 & $2.13 \times 10^{3}$ & 1.0 \\
Stycast & 300 & $1.18 \times 10^{3}$ & 0.26 \\
He $(100$ Torr $)$ & 5411 & 0.658 & 0.016
\end{tabular}

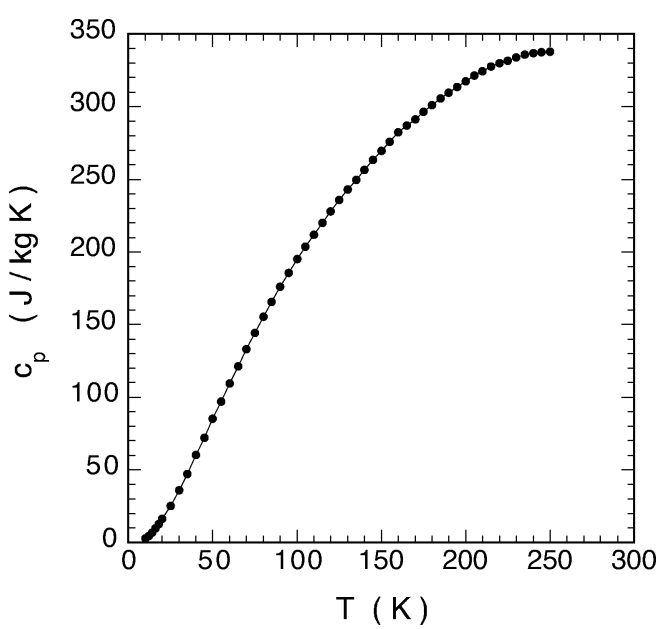

Fig. 2. Temperature dependence of $c_{p}$ for BSCCO. The unit was converted to the SI unit from the original data by Loram et al. [13].

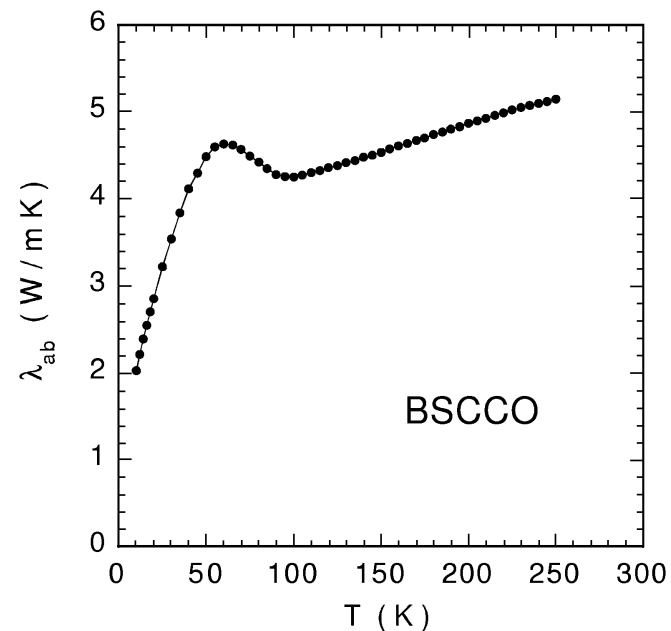

Fig. 3. Temperature dependence of the in-plane thermal conductivity $\lambda_{a b}$ for BSCCO. The data was converted and interpolated from the original data by Crommie and Zettl [14].

Since an electrical contact resistivity of less than $1 \times 10^{-6} \Omega \mathrm{cm}^{2}$ is realizable for the Au electrode, the thermal contact resistivity is reduced significantly in that case. Taking into account the very small $\lambda_{c}$ in addition, we neglected the thermal contact resistivity in the present model.

We are concerned with the transient response on a time scale of $100 \mathrm{~ns}$ to $1 \mu \mathrm{s}$. Since the velocity of He gas is of the order of $0.1 \mathrm{~m} / \mathrm{s}$, the second and the third term of (1) are negligible on this 


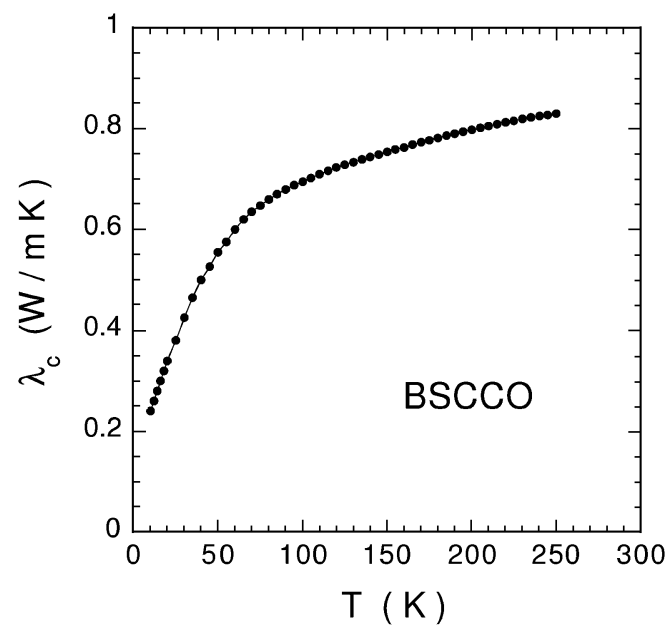

Fig. 4. Temperature dependence of the out-of-plane thermal conductivity $\lambda_{c}$ for BSCCO. The data was converted and interpolated from the original data by Crommie and Zettl [14].

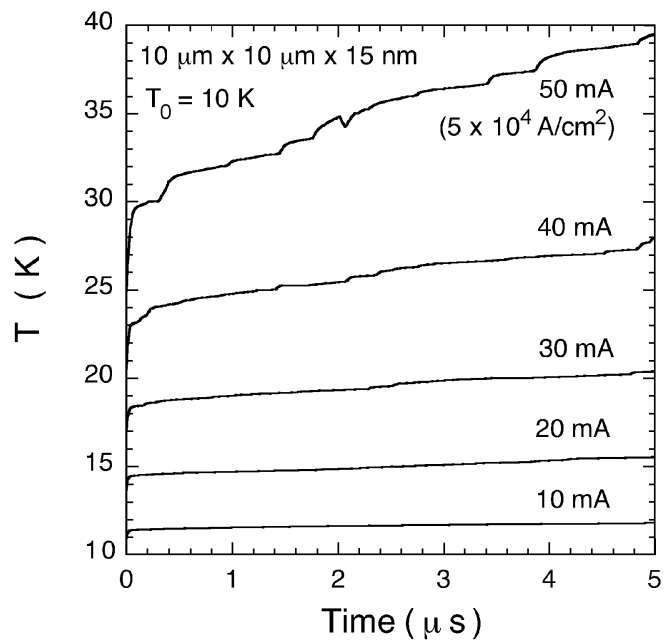

Fig. 5. Transient response of the mesa temperature at $10 \mathrm{~K}$ on a $5 \mu$ s time scale for a mesa $10 \mu \mathrm{m}$ wide and $15 \mathrm{~nm}$ thick with an injection quasiparticle current of $10 \mathrm{~mA}$ to $50 \mathrm{~mA}$.

time scale. At $10 \mathrm{~K}$, the mean heat diffusion length $\sqrt{\lambda t / c_{p} \rho}$ for $\mathrm{Au}$ is $32.5 \mu \mathrm{m}$ at $t=100 \mathrm{~ns}$ and $103 \mu \mathrm{m}$ at $1 \mu \mathrm{s}$, so that only the area reached from the mesa within this distance is heated. This is confirmed by the present numerical calculation results, in which the distant parts remain cooled. This means that the cell size should be less than a few $\mu \mathrm{m}$. In the numerical calculation, this size poses a constraint on the time division to be less $10^{-10} \mathrm{~s}$, since $\lambda \Delta t / c_{p} \rho \Delta x^{2}<1 / 6$ for the convergence of calculation in the three-dimensional case. In the mesa region, in which the cell size is far smaller, $\Delta t$ should be less than $5 \times 10^{-14} \mathrm{~s}$ for the same reason.

\section{RESULTS AND DISCUSSION}

The effect of He gas flow is found to be insignificant-very little heat flows into the He gas. The decrease in the temperature rise in the presence of the $\mathrm{He}$ gas is less than $1 \mathrm{~K}$ when the total temperature rise in its absence is $10 \mathrm{~K}$ to $20 \mathrm{~K}$.

Fig. 5 shows the numerical calculation results when a quasiparticle current $I$ of $10 \mathrm{~mA}$ to $50 \mathrm{~mA}$ is injected to a mesa of

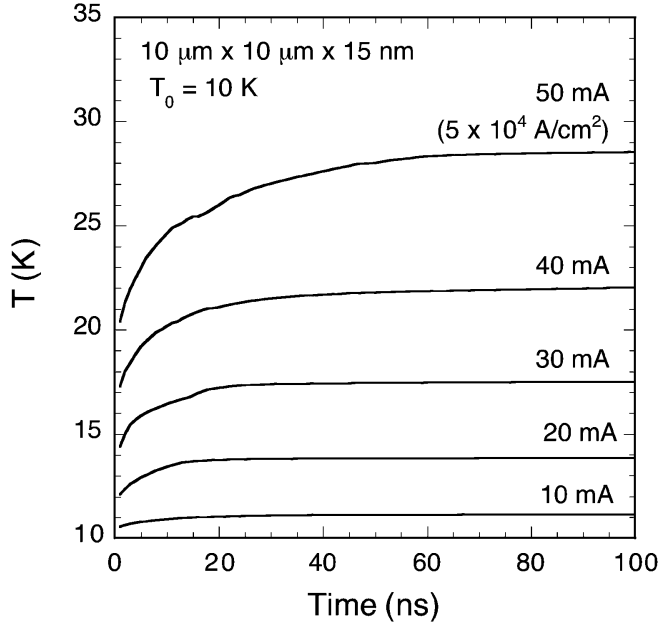

Fig. 6. Transient response of the mesa temperature at $10 \mathrm{~K}$ for a mesa $10 \mu \mathrm{m}$ wide and $15 \mathrm{~nm}$ thick with an injection quasiparticle current of $10 \mathrm{~mA}$ to $50 \mathrm{~mA}$ on a $100 \mathrm{~ns}$ time scale.

$10 \mu \mathrm{m}$ on a side and $15 \mathrm{~nm}$ in thickness at a base temperature of $T_{0}$ of $10 \mathrm{~K}$. The resistivity of BSCCO in the $c$-axis direction is $10 \Omega \mathrm{cm}$ at all temperatures. A typical critical Josephson current density is $10^{3} \mathrm{~A} / \mathrm{cm}^{2}$ for slightly overdoped BSCCO. It is seen that the temperature rises very rapidly at first and then it increases at a rate of $\sim 0.2 \mathrm{~K} / \mu$ s when $I=20 \mathrm{~mA}(2 \times$ $\left.10^{4} \mathrm{~A} / \mathrm{cm}^{2}\right)$. When the injection current is increased, the rate of temperature rise after the initial step increases. The initial rapid rise is due to the very small specific heat for BSCCO at this low temperature. The following slower temperature rise is due to the heat diffusion in the Au electrode. Similar numerical results were obtained by Fenton et al. [8].

Fig. 6 shows the temperature rise of the mesa on a $100 \mathrm{~ns}$ time scale, which is used for the short-pulse tunneling spectroscopy [12]. It is seen that the temperature rise at $60 \mathrm{~ns}$ is $3.5 \mathrm{~K}$ when $I=20 \mathrm{~mA}$, and $18 \mathrm{~K}$ when $I=50 \mathrm{~mA}$. This is compared with the short-pulse ITS for a slightly overdoped BSCCO, in which $I=20 \mathrm{~mA}$ nearly corresponds to the superconducting gap voltage and the $I=50 \mathrm{~mA}$ nearly corresponds to the edge of the voltage range. Therefore, the present numerical calculation indicates that in the short-pulse ITS measurements the temperature is raised 3 to $5 \mathrm{~K}$ at the superconducting gap voltage and 15 to $20 \mathrm{~K}$ near the edge of the voltage range, where the pseudogap is observed [6].

Fig. 7 shows the transient response of the temperature for small mesas with various sizes. When the mesa is $1 \mu \mathrm{m}$ in square size with the same injection current density of $2 \times 10^{4} \mathrm{~A} / \mathrm{cm}^{2}$, the temperature rise is approximately $1.2 \mathrm{~K}$ both at 60 and 500 ns. This implies that the reduction of the mesa size leads to the suppression of the self-heating to a great extent. This is a natural consequence from the fact that the temperature rise is substantially determined by the temperature of the Au electrode on top of the mesa within a thermal diffusion length. The area of $\mathrm{Au}$ to be heated is spanned mostly by the thermal diffusion length, while the heat elevating the temperature of the Au area is reduced proportionally to the square of the mesa size. Therefore, the temperature rise for smaller mesas is greatly suppressed. The 


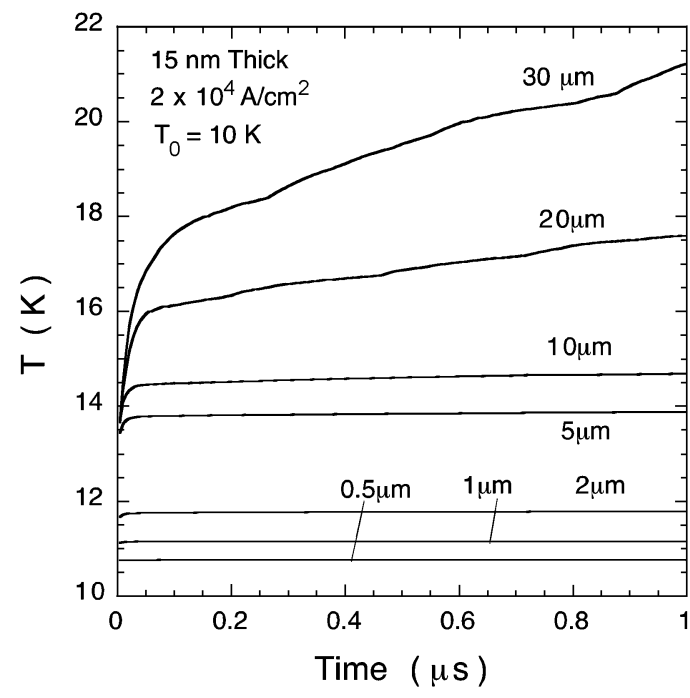

Fig. 7. Transient response of the mesa temperature at $10 \mathrm{~K}$ on a $1 \mu$ s time scale at an injection quasiparticle current density of $2 \times 10^{4} \mathrm{~A} / \mathrm{cm}^{2}$ for small square mesas with various edge sizes from $0.5 \mu \mathrm{m}$ to $30 \mu \mathrm{m}$ in side width with a 15 nm thickness.

importance of the electrode was also indicated by Krasnov et al. [9].

At an elevated base temperature $T_{0}$, the thermal conductivity of Au decreases, whereas the specific heat of BSCCO increases by a factor of orders of magnitude. Therefore, the relaxation time of the initial temperature rise becomes longer and the shortpulse technique becomes effective at elevated temperatures. For example, at $50 \mathrm{~K}$, a quasiparticle injection of $20 \mathrm{~mA}$ causes a temperature rise of $8.3 \mathrm{~K}$ at $5 \mu$ s while it is $3.3 \mathrm{~K}$ at $60 \mathrm{~ns}$.

The present result also implies that the temperature rise is much reduced in the ITS for underdoped samples, in which the current injection can be reduced at a fixed voltage due to high resistivity.

\section{CONCLUSION}

From the detailed numerical calculation of the self-heating in a small mesa structure, it is found that the temperature rise of a $10 \mu \mathrm{m}$ square $10 \mathrm{IJJ}$ stack mesa amounts to 4 to $6 \mathrm{~K}$ at a quasiparticle injection current of $20 \mathrm{~mA}$ and 18 to $30 \mathrm{~K}$ at $50 \mathrm{~mA}$ even in a short period of $100 \mathrm{~ns}$ to $5 \mu \mathrm{s}$. This implies that in the short-pulse interlayer tunneling spectroscopy experiments, the influence of self-heating on the superconducting gap magnitude for a slightly overdoped BSCCO is little, but it is significant in a voltage range much higher than the superconducting gap voltage. The influence is significantly reduced when the mesa is composed from an underdoped BSCCO or when the mesa size is reduced to a few $\mu \mathrm{m}$.

\section{REFERENCES}

[1] R. Kleiner, F. Steinmeyer, G. Kunkel, and P. Müller, "Intrinsic Josephson effects in $\mathrm{Bi}_{2} \mathrm{Sr}_{2} \mathrm{CaCu}_{2} \mathrm{O}_{8+\delta}$ single crystals," Phys. Rev. Lett., vol. 68, no. 15, pp. 2394-2397, Apr. 1992.

[2] R. Kleiner and P. Müller, "Intrinsic Josephson effects in high- $T_{c}$ superconductors," Phys. Rev. B, vol. 49, no. 2, pp. 1341-2394, Jan. 1994.

[3] A. A. Yurgens, "Intrinsic Josephson junctions: Recent developments," Supercond. Sci. Technol., vol. 13, no. 8, pp. R85-R100, Aug. 2000.

[4] M. Suzuki, T. Watanabe, and A. Matsuda, "Short pulse tunneling measurements of the intrinsic Josephson junction in $\mathrm{Bi}-\mathrm{Sr}-\mathrm{Ca}-\mathrm{Cu}-\mathrm{O}, "$ IEEE Trans. Appl. Supercond., vol. 9, no. 2, pp. 4507-4510, June 1999.

[5] M. Suzuki, T. Watanabe, and A. Matsuda, "Interlayer tunneling spectroscopy for slightly overdoped $\mathrm{Bi}_{2} \mathrm{Sr}_{2} \mathrm{CaCu}_{2} \mathrm{O}_{8+\delta}$," Phys. Rev. Lett., vol. 86, no. 26, pp. 5361-5364, June 1999.

[6] M. Suzuki and T. Watanabe, "Discriminating the superconducting gap from the pseudogap in $\mathrm{Bi}_{2} \mathrm{Sr}_{2} \mathrm{CaCu}_{2} \mathrm{O}_{8+\delta}$ by interlayer tunneling spectroscopy," Phys. Rev. Lett., vol. 85, no. 22, pp. 4787-4790, Nov. 2000.

[7] J. C. Fenton, P. J. Thomas, G. Yang, and C. E. Gough, "System for fast rime-resolved measurements of $c$-axis quasiparitcle conductivity in intrinsic josephson junctions of $\mathrm{Bi}_{2} \mathrm{Sr}_{2} \mathrm{CaCu}_{2} \mathrm{O}_{8+\delta}$," Appl. Phys. Lett., vol. 80, no. 14, pp. 2535-2537, Apr. 2002.

[8] J. C. Fenton and C. E. Gough, "Heating in mesa structures," J. Appl. Phys., vol. 94, no. 7, pp. 4665-4669, Oct. 2003.

[9] V. M. Krasnov, A. Yurgens, D. Winkler, and P. Delsing, "Self-heating in small mesa structures," J. Appl. Phys., vol. 89, no. 10, pp. 5578-5580, May 2000.

[10] V. N. Zavaritsky, "Joule heating versus 'intrinsic' tunneling in HTSC," Physica C, vol. 404, no. 4, pp. 440-443, May 2004.

[11] T. Hamatani, K. Anagawa, T. Watanabe, and M. Suzuki, "Extended-range short-pulse interlayer tunneling spectroscopy for $\mathrm{Bi}_{2} \mathrm{Sr}_{2} \mathrm{CaCu}_{2} \mathrm{O}_{8+\delta}$ intrinsic Josephson junctions," Physica $C$, vol. 390, no. 2, pp. 89-94, June 2003.

[12] K. Anagawa, Y. Yamada, T. Shibauchi, M. Suzuki, and T. Watanabe, "60 ns time scale short pulse interlayer tunneling spectroscopy for $\mathrm{Bi}_{2} \mathrm{Sr}_{2} \mathrm{CaCu}_{2} \mathrm{O}_{8+\delta}$," Appl. Phys. Lett., vol. 83, no. 12, pp. 2381-2383, Sept. 2003.

[13] J. W. Loram, J. L. Luo, J. R. Cooper, W. Y. Liang, and J. L. Tallon, "The condensation energy and pseudogap energy scale of BI:2212 from the electronic specific heat," Physica C, vol. 341-348, no. 2, pp. 831-834, Nov. 2000.

[14] M. F. Crommie and A. Zettl, "Thermal-conductivity anisotropy of single-crystal $\mathrm{Bi}_{2} \mathrm{Sr}_{2} \mathrm{CaCu}_{2} \mathrm{O}_{8}$," Phys. Rev. $B$, vol. 43, no. 1, pp. 408-412, Jan. 1991. 